\title{
Providing quality-of-service for frequency-aware Wi-Fi using OFDM-based variable-length Bloom filters
}

\author{
Suchul Lee ${ }^{1}$, Jaehyuk Choi ${ }^{2}$, Joon Yoo ${ }^{2}$ and Chong-Kwon Kim ${ }^{*}$
}

\begin{abstract}
Modern Wi-Fi networks are trending towards using a wider channel bandwidth to achieve high physical layer data rate. The wide channel band experiences fluctuations across the different frequencies, causing diversity in the frequency domain. Frequency-aware Wi-Fi protocols exploit this frequency diversity and consequently achieve high wireless capacity. However, most of the existing works have not considered quality-of-service (QoS) issues. In this paper, we present a new Wi-Fi protocol called QoS-Fi, that provides QoS for the mobile users in the frequency aware Wi-Fi network. QoS-Fi dynamically assigns orthogonal frequency division multiplexing (OFDM) subchannels for heterogeneous mobile users to meet the QoS demands. To achieve this goal, we apply an OFDM-based variable-length Bloom filter (VBF) that synergistically integrates the channel quality estimation and QoS channel coordination. To the best of our knowledge, this is the first work that employs QoS at the frequency domain for Wi-Fi networks. We study the impact of variable-length signatures in the aspect of throughput maximization and meeting the QoS requirements and further develop a decentralized QoS-aware channel-allocation algorithm that achieves sub-optimal performance. Our USRP/GNURadio-based experiments and trace-driven simulations show that QoS-Fi provides up to $1.39 \times$ and $1.29 \times$ throughput improvements compared to the legacy EDCA and well-known Knopp and Humblet's and round robin (K\&H/RR) scheduling, respectively in the QoS-regimes.
\end{abstract}

Keywords: Diversity; Wi-Fi; Variable-length Bloom filter; QoS provisioning; PHY/MAC protocols

\section{Introduction}

The recent development of communication technologies has enabled Wi-Fi networks to achieve high physical data rates. A consistent theme in Wi-Fi networks to advance the wireless capacity has been wider channel bandwidths as well as more antennas. The signals transmitted over a wide frequency band, however, experience independent fluctuations across different frequencies at a time instant. This phenomenon is generally called frequency-selective fading [1]. The modern wireless systems such as WiMAX [2] and 3GPP LTE [3] actually exploit this frequency diversity as another dimension of multi-user scheduling, by using the well-known orthogonal frequency division multiple access (OFDMA) technique. Here, the base station (BS) allocates each subchannel to the mobile user who has

${ }^{*}$ Correspondence: ckim@snu.ac.kr

1 Department of Computer Science, Seoul National University, Seoul, Korea Full list of author information is available at the end of the article better channel quality, consequently achieving near the Shannon's maximum capacity of wireless channels. Further, the BS can easily employ quality-of-service (QoS) strategies to the scheduler since the mobile users are tightly synchronized with the BS.

Several Wi-Fi protocols have been proposed [4-7] to harness the frequency diversity with a similar trend towards the use of wider channels. However, the Wi-Fi network renders several new challenges. First, it requires fine-grained channel estimation which incurs high channel estimation overhead [2-6,8,9]. Channel estimation in Wi-Fi depends on training sequences (dedicated pilots) or RTS/CTS-like probing frames, which consumes time and frequency resource. Second, the channel access requests from stations (STAs) should be coordinated, further inducing corresponding protocol overhead [4-6,10]. Third, modern Wi-Fi networks host diverse applications which render various QoS demands; some require small

\section{是 Springer}

(c) 2014 Lee et al. licensee Springer. This is an Open Access article distributed under the terms of the Creative Commons Attribution License (http://creativecommons.org/licenses/by/2.0), which permits unrestricted use, distribution, and reproduction in any medium, provided the original work is properly credited. 
latency while others need large throughput. It is challenging to be both aware of the frequency diversity and meet these QoS demands for real-time applications in Wi-Fi networks.

Several approaches have been proposed in Wi-Fi networks to achieve the frequency diversity awareness. Some work mainly focus on harnessing frequency diversity with additional channel estimation costs [4-6,10], while others improve protocol efficiency (e.g., reducing channel contention cost) by operating at the frequency domain [11-13]. Our previous work [7] proposes the Wi-Fi protocol that achieves the above objectives by using an OFDMbased Bloom filter. These schemes generally achieve high wireless capacity via frequency diversity awareness. However, none of the aforementioned schemes support the prioritized channel access of STAs for real-time traffic, thus cannot be applied to QoS-supported Wi-Fi networks.

In this paper, we present QoS-Fi, a Wi-Fi PHY/MAC protocol that exploits frequency diversity to satisfy the diverse QoS requirements of heterogeneous realtime mobile users. QoS-Fi dynamically assigns OFDM subchannels to heterogeneous real-time mobile users depending on their QoS requirements. To achieve this goal, we apply an OFDM-based variable-length Bloom filter (VBF) [14] that synergistically integrates two operations: (i) the channel quality estimation for exploiting frequency diversity and (ii) the frequency-aware channel coordination to meet the QoS requirements. To the best of our knowledge, this is the first work that provides $\mathrm{QoS}$ in the frequency domain for frequency-aware Wi-Fi networks.

The main features of the QoS-Fi protocol are as follows:

- QoS-Fi estimates the channel quality while concurrently performing the prioritized channel contention and coordination based on the OFDM-based Bloom filter. The QoS-Fi MAC protocol exchanges RTS/CTS-like QoS-collision resolution request $(\mathrm{Q}-\mathrm{CRQ}) / \mathrm{QoS}$-collision resolution reply (Q-CRP) frames through the VBF. Multiple STAs contend for OFDM subchannels simultaneously, according to estimated subchannel quality and QoS demand. The AP estimates the uplink channel qualities by using this synthesized Q-CRQ frame without any further channel estimation overhead. After the AP makes a decision for the subchannel allocation based on the channel qualities and QoS demands, it broadcasts the Q-CRP frame to inform the STAs of the coordination result.

- QoS-Fi supports the prioritized channel access by using variable length signatures in the VBF. The STA who participates the channel contention with a longer signature has a larger channel access probability than the STA with a shorter length signature. This mechanism resembles the EDCA in 802.11e in the aspects that, by using various arbitrary interframe spaces (AIFSes), high priority traffic has higher channel access and lower collision probabilities. We study the impact of the use of variable length signatures in our protocol by mathematical analysis and simulations. The results reveal that QoS-Fi maximizes the throughput while supporting the diverse QoS requirements with small amount of overhead.

In summary, this paper makes the following contributions. (i) We design and implement QoS-Fi, a novel WiFi PHY/MAC protocol that exploits frequency diversity and supports QoS demands, while achieving PHY/MAC efficiency. (ii) We study the impact of the length of signatures in the aspect of both service differentiation and false positive probability of the Bloom filter. We apply VBF to the QoS-Fi protocol to perform the prioritized channel contention and coordination. (iii) We prototype QoS-Fi on the universal software radio peripheral (USRP)/GNURadio platform and evaluate its performance using the detailed trace-driven simulation. Our results show that QoS-Fi provides up to $1.39 \times$ and $1.29 \times$ throughput improvements compared to the legacy EDCA and well-known Knopp and Humblet's and round robin (K\&H/RR) scheduling, respectively, while providing the prioritized channel access in the QoS regimes.

The rest of this paper proceeds as follows. Section 2 reviews the related work, and Section 3 describes the detailed design of the QoS-Fi PHY/MAC protocol. Then, Section 4 presents the channel coordination algorithms for QoS provisioning. In Section 5, we provide a detailed analysis to examine the impact of the length of signatures in the VBF. Section 6 evaluates the QoS-Fi's performance using our experimentation and trace-driven simulation, and finally, Section 7 concludes the paper.

\section{Related work}

\subsection{Improving MAC efficiency}

The general Wi-Fi MAC protocols [8] employ the CSMA/CA exponential backoff algorithm, which is conducted in the time domain. In contrast, the frequency domain contention protocols $[7,11-13,15]$ have been proved to be much more efficient in terms of wireless capacity. FICA [11] redesigns the PHY/MAC by using an OFDM-based fine-grained channelization to attain protocol efficiency. Similar to FICA, [12] proposes Back2F that migrates the time domain backoff to the frequency domain. As the frequency domain backoff lasts for only several OFDM symbols, it reduces the contention time. REPICK [13] modifies the receiver to conduct frequency domain backoff instead of a transmitter and added a ACK piggybacking feature. These work share the similarity of 
using the OFDM technique to enhance the MAC protocol efficiency. Our previous work, D-Fi [7], exploits the frequency diversity while reducing overhead by using the Bloom filter-based channel contention and estimation. This paper further extends D-Fi by adding QoS provisioning functionalities that enable the prioritized channel access by leveraging the variable-length Bloom filter.

\subsection{Frequency diversity}

Many theoretic classic proposals exploiting frequency diversity are summarized in the wireless communication textbook [1]. Some of them are widely used by cellular systems such as mobile WiMAX [2] and 3GPP LTE [3]. Recently, there are theoretic studies that apply proportional fair packet scheduling in FDMA-based 3GPP LTE $[16,17]$ and CSMA-based OFDMA systems $[10,18]$. These work solved the resource allocation problem by mathematical modeling while assuming that the perfect channel quality information is available via the training sequence (e.g., pilot signal). This is less practical in Wi-Fi networks, since the AP and users do not maintain tight synchronization as in the cellular system. Therefore, we practically consider the Wi-Fi channel estimation overhead.

Many practical measurement studies have been conducted to show the existence of frequency diversity. Among these, the most relevant to our work are measurement studies in the $2.4 \mathrm{GHz} / 5 \mathrm{GHz}$ ISM bands $[4,6,19,20]$. Also, in wireless local area networks (WLANs), several frequency diversity-aware schemes have been proposed [4-6]. The authors of [4] observed the frequency diversity in wide-band WLANs and introduced a practical rate adaptation scheme based on the effective SNR (eSNR). In FARA [5], a transmitter can send multiple packets to multiple receivers concurrently based on the OFDM technique. Thus, it does not need to consider the time-sync problem arisen when multiple packets are combined at a receiver. FARA can be used at the downlink WLAN and may be viewed complementary to our work, since we mainly focus on the uplink. Finally, the authors of [6] proposed a diversity-aware WLAN that uses an adaptive interleaver and a forward error correction (FEC) scheme based on per-subcarrier channel state information (CSI). It adopts different domain approaches such as a persubcarrier FEC method and an interleaver and hence is orthogonal to our work.

\subsection{QoS-provisioning}

QoS provisioning in wireless networks has been widely studied from different perspectives. We classify them into two categories: centralized approaches and decentralized approaches. The centralized approaches generally involve admission control and scheduling. For example, the main idea of resource allocation in [21] is to compute the reserved bandwidth for each user in terms of the average delay. In [22], the authors proposed a powerful concept termed effective capacity, which enables to analyze the statistical delay. IEEE 802.11e [23] standard defines EDCA, a decentralized QoS support protocol. Unlike centralized QoS provisioning schemes, the EDCA mechanism does not require a dedicated central entity that computes computationally complex scheduling and/or admission control algorithms. As a result, the EDCA mechanism only supports a certain level of QoS differentiation as opposed to the centralized approaches that provides QoS guarantees. Our QoS-Fi protocol incorporates two features from each type of the QoS provisioning mechanism: (i) the EDCA-like decentralized feature for being applied in WiFi networks and (ii) the admission control algorithm that enhances QoS provision functionality using the effective capacity concept [22].

\subsection{Bloom filter}

The Bloom filter is a space-efficient data structure. Here, the space applies to the network resources, which usually directly affects the protocol efficiency. The price paid for this space efficiency is the probabilistic ambiguity inherent to the Bloom filter: it tells us that the element either definitely is not in the set or may be in the set. This space efficiency enables the Bloom filter to be utilized in many network-related applications, some representative examples are summarized in [24]. Our previous work [7] shows that the OFDM-based Bloom filter implementation is practically feasible in real Wi-Fi networks. It mainly deals with the ambiguity problem raised from the use of Bloom filter and addresses the problem. There are many Bloom filter variants, including variable length Bloom filter, counting Bloom filter, deletable Bloom filter, etc [25]. Among them, in this paper, we apply the variable length Bloom filter [14] to augment the QoS provisioning functionality.

There are other methods that provide the similar functions, i.e., simultaneous channel coordination and estimation. For example, one can use pseudorandom noise sequence (PN) [26] or Zadoff-Chu sequence [27] (ZC one of the constant amplitude zero autocorrelation waveform, a.k.a., CAZAC) for the similar goal of QoS-Fi. Though the sequence-based method can provide more accurate channel estimation, generally, it incurs larger overhead. While superimposing multiple sequences can mitigate the overhead, it decreases channel estimation accuracy. In addition, the sequence-based channel estimation generally suffers from the so-called 'estimation error floor' problem, where the error does not depend on channel noise itself but the length of sequences. Finally, the Bloom filter can also produce more accurate channel estimation, by using the cross correlation technique like PN sequences. However, it naturally incurs larger overhead compared to Bloom filter (as Q-CRQ/Q-CRP is 
implemented with an OFDM symbol) and suffers from the same problems mentioned so far.

\section{QoS-Fi protocol design}

In this section, we first present the overview of the proposed protocol and then give details of the PHY/MAC design.

\subsection{Protocol overview}

QoS-Fi is an OFDM-based QoS-aware PHY/MAC protocol that allows different Wi-Fi STAs to access several narrower orthogonal subchannels with differentiated channel access priority in a wide-band Wi-Fi. Figure 1 illustrates the basic channel access scheme for QoS-Fi. In QoS-Fi, a wide-band Wi-Fi band is divided into several orthogonal subchannels and each of them is used as a channel access unit. QoS-Fi STAs select multiple candidate subchannels and contend for the use of the selected subchannels based on their traffic demands and QoS requirements. The channel access for each subchannel is coordinated through RTS/CTS-like Q-CRQ/Q-CRP frames (Sections 3.2.2 and 3.2.3). If the medium is idle for the period of distributed interface space (DIFS), QoSFi STAs transmit Q-CRQ frames simultaneously on their selected subchannels, where each STA selects candidate subchannels likely to have good channel quality. Q-CRQ frames transmitted on each subchannel contain the transmission information of the corresponding STAs with a binary bit sequence called a signature.

Similar to the 802.11 EDCA, the de facto QoS MAC protocol for Wi-Fi, QoS-Fi defines four levels of priority for channel access by assigning four types of signatures, each of which corresponds to an access category (Section 3.2.1).

Thus, each STA has four different signatures and conveys one of them to the QoS-AP through Q-CRQ frames according to QoS requirements and the corresponding access categories (Section 3.2.1). Upon the receipt of QCRQs sent from multiple STAs, the QoS-Fi AP estimates the uplink channel quality of the STAs (Section 3.2.4) and performs frequency-aware subchannel allocation to the STAs based on a pre-defined channel allocation policy, for example, maximizing throughput subject to the QoS requirement of each user. Then, the AP broadcasts the channel allocation results to the QoS-STAs through a Q-CRP frame (Section 3.2.3). The Q-CRP frame conveys the signature of the contention winner and transmission rates for future data transmission. When a subchannel is selected by multiple STAs, Q-CRQ frames from the STAs on the subchannel are collided at the AP. The QoS-Fi uses the OFDM-based Bloom filter technique in conjunction with prioritized signatures of variable length to resolve the channel contention for a subchannel, where the Q-CRP frames with longer signature lengths are selected with higher probability, thus achieving the prioritized channel access (Section 3.2).

\subsection{Channel contention and estimation with QoS-provisioning}

\subsubsection{Signatures with QoS-provisioning}

Signature. A signature is a binary bit sequence of 64 bits, which is uniquely assigned to an individual QoS-Fi STA by a QoS-Fi AP when the STA associates to the AP, where the AP manages a set of the assigned signatures $S=\left\{s_{1}, s_{2}, \ldots, s_{N}\right\}$. The the number of bits $l$ marked as ' 1 ' in the signature is defined as the length of the signature $l$.

In QoS-Fi, the QoS provisioning mechanism is implemented by using variable length signatures, where the longer length a signature has, the higher a QoS priority it becomes. In particular, similar to 802.11e EDCA, QoS-Fi uses four signature lengths $l_{1}>l_{2}>l_{3}>l_{4}$ to define four levels of the priority. Hence, every STA joining the network is assigned four unique signatures, each of which corresponds to one of the four access categories. The STA contends for wireless subchannels with Q-CRQ frames in which one of the four signatures is embedded based on the priority of the traffic from the upper layer.

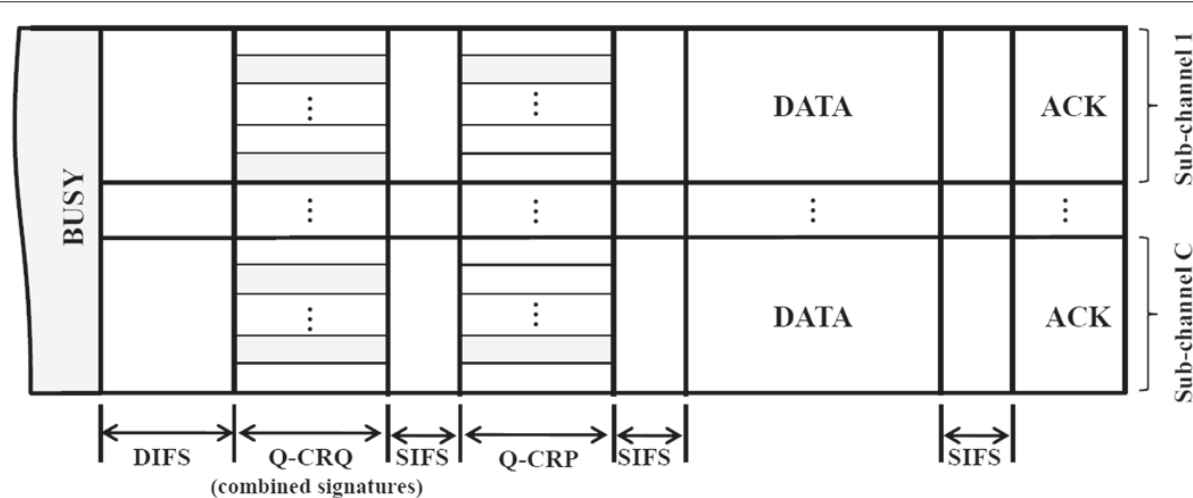

Figure 1 The QoS-Fi MAC protocol overview [7]. 
Signature generation. The QoS-Fi AP uses hash functions $^{\text {a }}$ to generate a signature of length $l_{i}, i \in\{1,2,3,4\}$. The hash value from a hash function is used as a pointer that specifies one of the 64 bits (i.e., position of a bit) and the selected bit is marked as 1 . A signature of length $l$ can be generated through the superposition of $l$ bit sequences of length 1 , i.e., the result of binary OR, where the $l$ bit sequences are generated by a certain set of hash functions ${ }^{\mathrm{b}}$. We use each STA's unique identifier, e.g., MAC address, as a search key to choose a set of hash functions from the hash function pool to generate a signature for the STA.

Implementing signatures in OFDM systems. A signature is carried over one subchannel via a Q-CRQ frame; 1 bit over one subcarrier. We use binary amplitude modulation (BAM) to modulate a single bit on each subchannel. BAM uses on-off signaling that maps a binary ' 0 ' to zero amplitude and a binary 1 to a random complex number on the unit circle $\left(e^{j \theta}\right)$ in a subcarrier. In other words, no signal is transmitted to modulate a binary 0 in a subcarrier and a fixed powered random complex signal is transmitted to modulate a binary 1 in a subcarrier. A receiver can easily detect a BAM symbol by measuring a signal power level on a subcarrier without demodulating an exact symbol.

\subsubsection{Channel contention with Q-CRQ frame}

Frequency-domain channel contention. In our previous work [7], we have introduced a frequency-aware channel access protocol, D-Fi. QoS-Fi follows the D-Fi's OFDM-based channel model and extends D-Fi's channel access protocol. QoS-Fi divides a Wi-Fi band into several orthogonal subchannels and each of them is used as a channel access unit, as shown in Figure 1. We choose 64 contiguous subcarriers for a subchannel, forming total 8 subchannels (bandwidth is $2.5 \mathrm{MHz}$.). Among 64 subcarriers, 60 subcarriers are used for data transmission and the rest 4 subcarriers are used as a pilot channel that tracks the subchannel quality while the data is being transferred (see [7] for the details).

Subchannel request. QoS-Fi STAs conduct channel contention in the frequency domain through RTS/CTSlike Q-CRQ/Q-CRP frames. Each QoS-Fi STA selects multiple candidate subchannels, in particular $K$ subchannels, and contends for the selected subchannels by transmitting Q-CRQ frames over $K$ subchannels simultaneously. Upon the receipt of CRQs sent from multiple STAs, the QoS-Fi AP estimates the uplink channel quality of the STAs and performs subchannel allocation to the STAs based on a predefiend channel allocation policy, for example, proportional fairness or throughput optimum.

One may inquire how much the Q-CRQ/Q-CRPbased channel contention is efficient, when compared to the legacy binary exponential backoff (BEB)-based one.
Table 1 shows the timing parameters of QoS-Fi and $802.11 \mathrm{n}$. Assuming a 1,500-byte packet and the MCS of $54 \mathrm{Mbps}, 210 \mu \mathrm{s}$ is required to transmit a single packet. This is computed by using the smallest overhead that the legacy 802.11 produces. If more faster MCS schemes are used or smaller packets are transmitted, the gain of the Q-CRQ/Q-CRP based channel contention over the BEBbased one will be increased. Note that the longer cyclic prefix $(\mathrm{CP})$ is required to compensate the propagation delay of stations for the Q-CRQ symbol.

QoS request in Q-CRQ frames. Each QoS-Fi STA has four unique signatures of length $l_{i}, i \in\{1,2,3,4\}$ and performs channel contention by conveying one of the signatures to the AP via Q-CRQ frames according to the QoS level of the current traffic. For instance, when the QoS-Fi STA has traffic of the highest priority, e.g., VoIP, it transmits a Q-CRQ frame with its longest signature of length $l_{1}$ on the selected $K$ subchannels. Since each QoS-Fi STA transmits Q-CRQ frames over $K$ subchannels simultaneously, multiple Q-CRQs sent from different STAs may be overlapped on a subchannel.

To resolve such simultaneous channel contention, QoSFi uses the Bloom filter [24]. As depicted in Figure 2a, we consider a subchannel over which one or more signatures are transmitted as a Bloom filter consisting of 64 bits and identify a STA by checking whether the signature assigned to the STA is present in the filter or not. If only one signature is transmitted over the subchannel, then the signature can be identified easily. If two or more signatures collide, the AP uses the Bloom filter technique to identify which signature(s) are present in the collision. The identified signatures inform the AP of the possible transmitters and their priority levels of the traffic. The process of identifying signatures from a Bloom filter is called 'Q-CRQ decoding' (Figure 2b).

Q-CRQ decoding. Figure $2 \mathrm{~b}$ depicts an example of the Q-CRQ decoding process on a subchannel for a scenario where a Bloom filter, an array of 64 bits, at the QoSFi AP is built from two signatures $s_{1}$ and $s_{2}$ sent from STA1 and STA2, respectively. To test if a signature $s_{i} \in$ $S, 1 \leq i \leq N$, is in the Bloom filter, the AP checks

Table 1 Timing parameters of QoS-Fi and 802.11n

\begin{tabular}{clcl}
\hline & QoS-Fi & $\mathbf{8 0 2 . 1 1 n}$ & Remarks \\
\hline Q-CRQ & $37.4 \mu \mathrm{s}$ & $\mathrm{N} / \mathrm{A}$ & Long CP \\
Q-CRP & $28.4 \mu \mathrm{s}$ & $\mathrm{N} / \mathrm{A}$ & Short CP \\
BEB & $\mathrm{N} / \mathrm{A}$ & $\geq 144 \mu \mathrm{s}$ & $t_{\text {slot }}=9 \mu \mathrm{s}$ and \\
& & $\mathrm{CW}_{\text {min }}=32$ \\
$\begin{array}{c}\text { Contention } \\
\text { cost }\end{array}$ & $90 \mu \mathrm{s}$ & $144 \mu \mathrm{s} \sim$ & \\
\hline
\end{tabular}


a

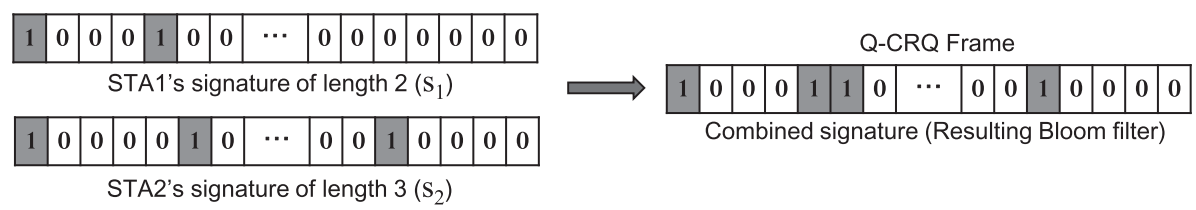

b

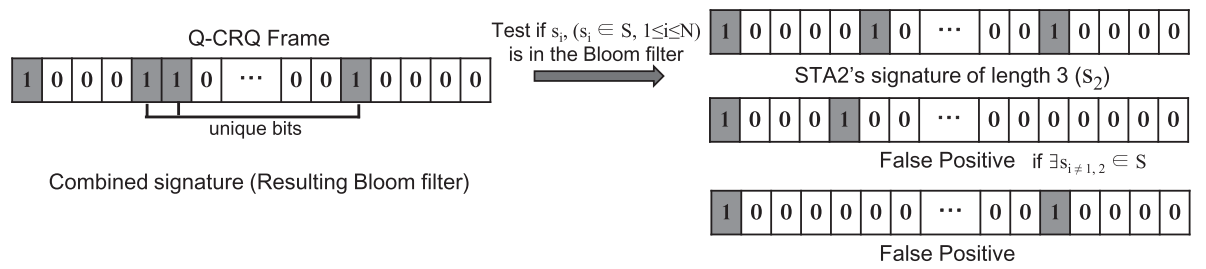

Figure 2 The Bloom filter-based operations. (a) The Q-CRQ transmissions procedure is described with the Bloom filter operations. (b) The Q-CRQ decoding process; only two false positives examples are depicted.

whether all the filter bits corresponding to all the bits set to 1 in $s_{i}$ are also marked as 1 . If not, then clearly $s_{i}$ is not a member of the filter and the AP considers that STA $i$ has not requested for the subchannel. If all corresponding bits are set to 1 , the AP assumes that $s_{i}$ is in the filter and STA $i$ has requested for the subchannel, although it may be a false positive case, i.e., the signature $s_{i}$ that actually is not present in the filter. In the example, only two false positive cases (of length 2) are presented while other longer false positive signatures can be possible.

Ambiguity problem of Bloom filter. In Q-CRQ decoding, we should handle two types of ambiguity: the physical and logical errors.

Physical errors. On the selected subchannels, a STA will transmit a signal over the OFDM subcarriers representing its signature. A single OFDM subcarrier should represent only one corresponding bit of a Bloom filter. However, since the frequency separation between subcarriers is imperfect in practice, a signal may spill over adjacent subcarriers, i.e., a subcarrier suffers from so-called 'spectral leakage' [28]. Furthermore, subcarrier-level signal detection is typically implemented by threshold-based decision approaches $[11,12]$ which compare the received signal power level with a predetermined threshold. As a result, signals can be falsely detected (or falsely missed). We call these two types of physical errors 'bitwise false positive (bitwise-FP)' and 'bitwise false negative (bitwiseFN)', respectively. We will show that this physical error problem can be mitigated effectively through an adaptive threshold adjustment technique in Section 6. Our implementation with a software radio platform (explained in Section 6) is shown to achieve very low physical error rate.

Logical errors. There can be 'false positive (FP)' cases during the Q-CRQ decoding process when using a Bloom filter. That is, the QoS-Fi AP may falsely decode the signatures and indicate the STAs that are not actually requested. For example, suppose that two $\mathrm{STAs}_{\mathrm{STA}}$ and $\mathrm{STA}_{2}$, whose signatures (of length 2 ) are ' $11000 \ldots 00$ ' and '01100...00', respectively, have requested the same subchannel, generating the Bloom filter of ' $1110 \ldots 00$ '. In this case, due to the inherent ambiguity, the AP may falsely indicate a signature of ' $10100 . . .00$ ' from a superposition of the two signatures ' $1110 \ldots 00$ ', which is neither $\mathrm{STA}_{1}$ 's nor $\mathrm{STA}_{2}$ 's .

To address the ambiguity problem of the Bloom filter, we analyze the FP probability with QoS-Fi protocol and derive the protocol parameter $K$ that minimizes the FP (Section 5), where QoS-Fi STAs request $K$ subchannels at once.

Prioritized channel access. The QoS-Fi AP uses the Bloom filter technique to identify the contending STAs for each subchannel as well as their QoS information. Recall that in QoS-Fi, the QoS provisioning mechanism is implemented by using variable length signatures, where the longer length a signature has, the higher a QoS priority it becomes. It is also worth mentioning that the Bloom filter decodes the longer signatures with the lower false positive rates when variable elements are mixed 
in the filter. Hence, the QoS-Fi AP identifies Q-CRQ frames with higher priorities (i.e., longer signatures) more accurately. This property is very important for service differentiation in QoS-Fi; it is straightforward that the false positive in the decoding process for a higher priority signature is more harmful than that of a lower priority signature since the higher priority STAs are allocated channels more frequently. This resembles the EDCA of $802.11 \mathrm{e}$ in the aspects that, by using various arbitration inter-frame spaces (AIFSes), high priority traffic has higher channel access and lower collision probabilities. However, the QoS-support in QoS-Fi is more stringent than EDCA because various channel allocation policies can be applied for high priority traffic by the QoS-Fi AP.

Consequently, when a STA has the most delay-sensitive, i.e., the highest priority level, traffic to transmit, it participates the channel contention with the longest signature among his own signatures. The STA who contends for the channel with a longer signature has a higher channel access probability than the STA with a shorter one.

\subsubsection{Channel assignment with Q-CRP Frame}

To inform STAs of subchannel allocation results, a QoS-Fi AP broadcasts a Q-CRP frame. The Q-CRP frame consists of two consecutive OFDM symbols: one for conveying the signature of a contention winner, and the other for data rate information for future data transmission, for each subchannel.

\subsubsection{Channel quality estimation}

During the $\mathrm{Q}-\mathrm{CRQ}$ decoding process, the AP can recognize that some bits marked as 1 in the filter are set by only one STA's signature, not overlapped by two or more another signatures. We refer to these bits as unique bits. After the Q-CRQ decoding, we exploit the unique bits to estimate the uplink channel quality of STAs. We employ a simple channel quality estimation method using the signal strength of the OFDM symbol. We assume that all STAs use the same transmission power and the total transmission energy spreads evenly over each of $l$ bits marked as 1 when sending a Q-CRQ symbol. Then, the AP uses the average energy level of the unique bits that belong to a signature as the channel quality of the STA that transmitted that signature (Figure 2).

We evaluate the performance of our channel estimation method in Figure 3. Despite its simplicity, the results show that, in most cases ( $\geq 90 \%$ ), the estimation error of our method is less than or equal to $1 \mathrm{~dB}$. Unlike channel state information (CSI) used in MIMO systems, this lightweight method, i.e., using the signal strength as channel quality, requires no complicated channel information exchange between an AP and STAs and thus is simply applicable to our system.

\section{Channel allocation with QoS provisioning}

There are two design issues while using the QoS-Fi protocol. The first revolves around the behavior of the QoS-Fi STAs that how each STA selects several subchannels for channel request. The second issue is how the QoS-AP allocates subchannels to the STAs so that it maximizes the overall throughput subject to the QoS requirements of each STA.

In this section, we present (i) the (decentralized) user-side algorithm which explores/exploits frequency diversity and (ii) the AP-side frequency-aware channel allocation algorithm that performs the channel assignment based on the channel quality estimates and the QoS requirement of each STA. We consider a scenario where $N$ STAs are conducting channel contention for $C$ subchannels.

\subsection{User-side distributed channel explore/exploit algorithm}

In QoS-Fi, each STA employs a simple distributed learning algorithm to adaptively select $K$ subchannels that are likely to have good channel quality, thus achieving throughput improvement. To this end, each STA maintains a state vector, $[x(1), x(2), \ldots, x(C)]$, where let $x(i)$ denote the preference factor for subchannel $i$ with $\sum_{i}^{C} x(i)=1$. All $x(i), i \in\{1,2,3, \ldots, C\}$, are initialized to $1 / C$.

Upon every receipt of Q-CRQs sent from multiple STAs, the QoS-Fi AP estimates the uplink channel quality of the STAs and informs STAs of channel allocation results for each subchannel by broadcasting Q-CRP frames over $C$ channels. Then, the STAs learn the channel allocation results from the Q-CRP frames, including whether they are granted subchannels or not. Note that if a STA is granted a requested subchannel, it implies that the channel has a good condition for the STA and vice versa. Based on the allocation result for each channel, each STA adjusts $x(\cdot)$ in an additive increase/multiplicative decrease (AIMD ${ }^{\mathrm{d}}$ ) manner. When the STA is granted subchannel $i$, the STA increases the value of $x(i)$ by $\alpha$. For non-selected subchannel $i$, the STA decreases the value of $x(i)$ multiplicatively by $1 / \beta$. The updated state vector, $[x(1), x(2), \ldots, x(C)]$, is then normalized so that their sum is to be 1 .

Algorithm 1 shows the pseudocode of the user-side channel exploration/exploitation algorithm. A STA selects total $K$ subchannels where subchannel $i$ is selected with the probability proportional to the weight $x_{i}$. Obviously, the optimal value of $K$ depends on the number of active STAs $(N)$ in a network. An AP estimates the number of 


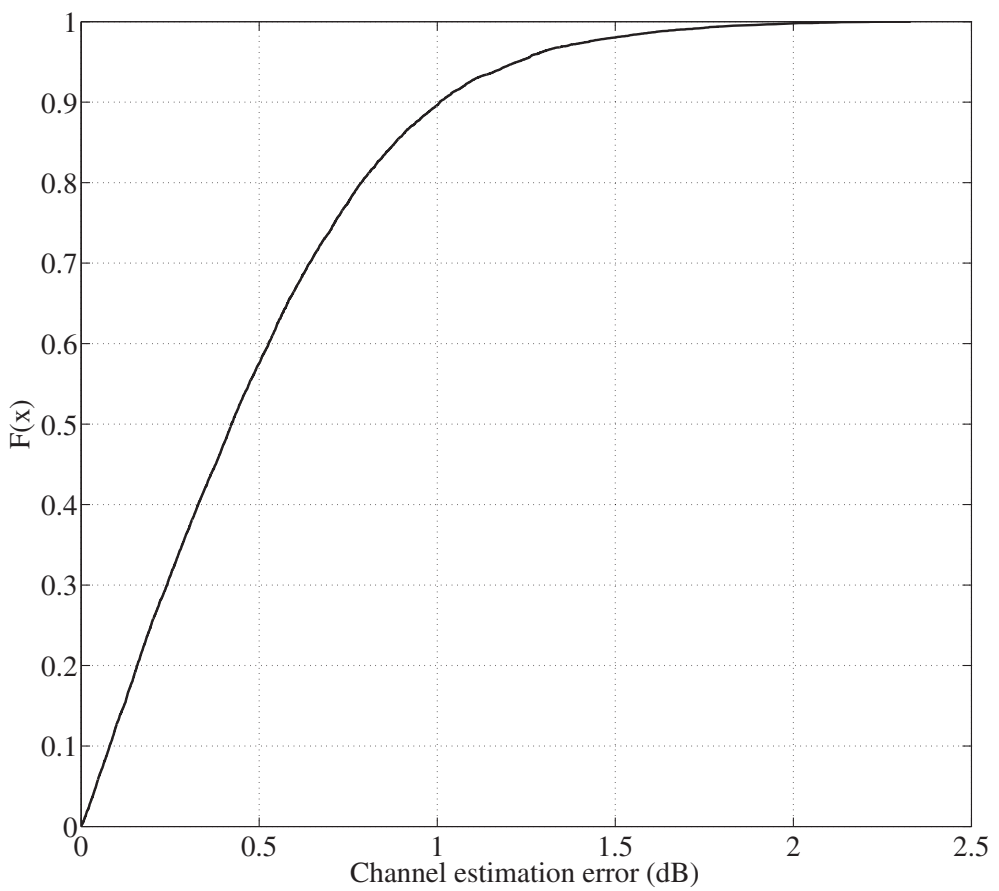

Figure 3 The experimental result: the empirical CDF for the estimation error of our method.

active STAs in the network [29] and periodically broadcasts an appropriate $K$ value. We adjust $K$ such that the false positive probability is not large (e.g., $\leq 10 \%$ ) based on the analysis shown in Section 5.

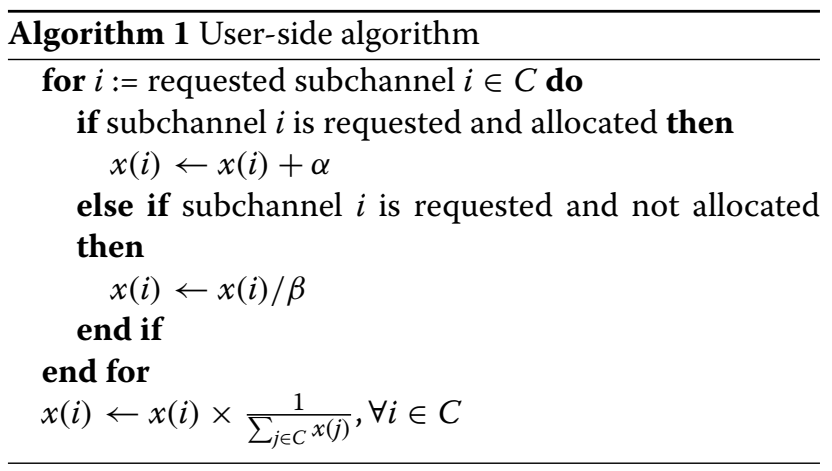

\subsection{AP-side algorithm}

Every time the QoS-Fi AP receives the requests from its associated STAs, the AP measures the channel quality of each STA and uses it to allocate the subchannels in such a way that maximizes the overall throughput subject to the QoS requirements of each STA. In particular, the AP-side channel allocation algorithm comprises two combined phases.

The first phase is the QoS provisioning phase that for a given combination of subchannel allocation, the QoS AP exams whether the allocation satisfies the QoS requirement of each STA's request or not. The AP utilizes an admission control algorithm for this test.

We assume that STA $i, i \in\{1,2, \ldots, N\}$ using subchannel $c, c \in\{1,2, \ldots, C\}$ in data transmission phase $t, t \in \mathbb{N}$ has a data rate $r_{i, c}(t)$ and a specified delay bound $D_{\max }$ (pre-defined with the QoS types). The delay experienced by the packet of STA $i$ transferred by subchannel $c$ in data transmission phase $t$ is $D_{i, c}(t)$ and is required to be less than or equal to the delay bound $D_{\max }$ for the QoS requirement (Equation 2).

In Equation 2, the delay needs to be estimated for each packet. Since multiple packets sent from multiple stations are transferred simultaneously, each data transmission phase lasts until the end of the longest packet transmission among multiple packets transferred in all the subchannels, as shown in Figure 1. Therefore, each packet in the same transmission phase will experience equal delay, which definitely depends on the future channel allocation decision.

Among a set of candidate allocation that satisfies the QoS requirement, the second phase selects the allocation that maximizes the sum rate transferred over all the subchannels, in the following data transmission phase. To relax the complexity of the problem, we assume that the total transmission power of station $i$ is equally divided among the subchannels assigned to the station.

Let $r_{i, c}(t)$ be the data rate of STA $i$ using subchannel $c$ in data transmission phase $t$. Then, the strategy of 
maximizing the sum rate subject to the QoS requirement of each STA is written as

$$
\begin{aligned}
& \max \sum_{i} \sum_{c} r_{i, c}(t), \\
& \text { subject to } D_{i, c}(t) \leq D_{\max } .
\end{aligned}
$$

The transmit rate of station $i$ using subchannel $c$ can be calculated according to Shannon's capacity process. Then, the sum rate can be further rewritten as a function of estimated SNRs, i.e., $\mathrm{SNR}_{i, c}$.

The challenges of this problem lie in its complexity. Obviously, our problem is an integer program (IP) and is proven to be strongly NP-hard [30], even with the assumption of the fixed power allocation. In addition, though the IP problem can be approximately solved with the wellknown LP relaxation technique [31] or the equivalent dual problem by using Lagrangian method [32], these methods still suffer from several difficulties to be applied in practice. For example, a subgradient algorithm that finds the solution of the dual problem converges too slowly to be useful. Thus, it cannot be applied to our QoS-Fi protocol because every channel contention is required to produce a quick channel allocation result (SIFS duration).

We then propose a more efficient sub-optimal solution with much lower complexity. It is shown that the channel allocation of QoS-Fi becomes close to the optimal performance.

Algorithm 2 shows the pseudocode of the AP-side channel allocation algorithm. Our AP-side algorithm is briefly summarized as follows: (i) it first determines the initial channel allocation without considering the QoS requirement of each station, (ii) computes the delay that STAs will experience in the followings data transmission phase based on the initial channel allocation, and (iii) according to the computed delay, cuts off the station that cannot meet the QoS requirement.

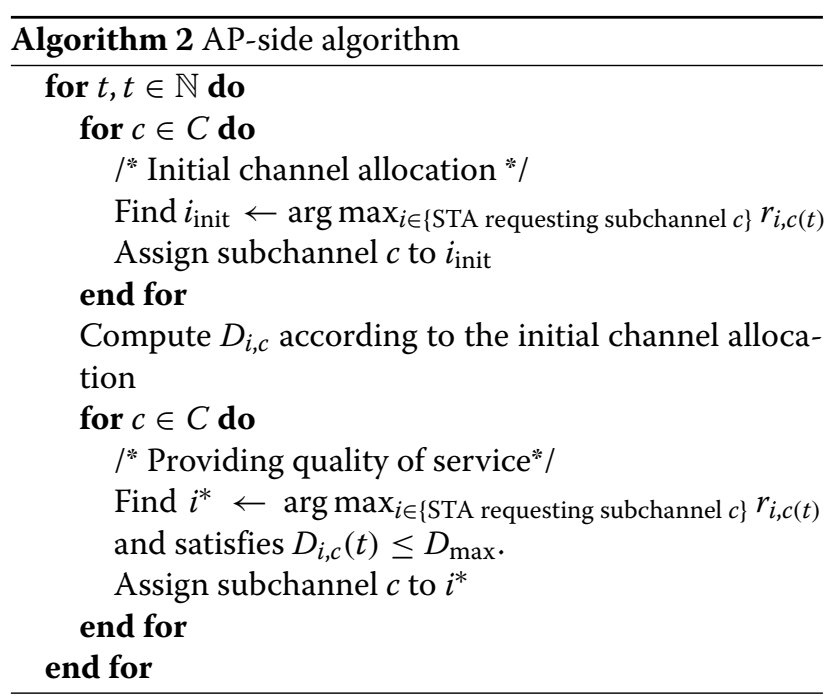

\section{Analysis of false positive probability}

In this section, we analyze the false positive probability in QoS-Fi.

\subsection{Network model}

We consider a scenario where $N$ STAs are conducting channel contention for $C$ subchannels by requesting $K$ subchannels $(K \leq C)$ repeatedly. QoS-Fi uses a separate Bloom filter for each subchannel, and thus, $C$ Bloom filters are generated every time the QoS-Fi STAs contend for $C$ subchannels, where a Bloom filter consists of $m$ binary bits (i.e., subcarriers). As explained in Section 3.2, the bits marked as 1 in each signature is uniformly distributed ${ }^{\mathrm{e}}$; thus, the probability that a certain bit is marked as 1 by a hash function is evenly fair over the bit sequence in a signature.

Let $r$ denote the average number of requests from the STAs for a subchannel. Then, $r$ elements (signatures) will be inserted into a Bloom filter. For example, assuming the uniformly distributed channel requests, i.e., each STA selects all subchannels with the same probability, $r$ is given by $\frac{N \times K}{C}$. Recall that for QoS support with four levels of prioritized channel acccess, each STA is assigned four (unique) signatures of length $l_{1}>\cdots>l_{4}$, where a longer signature implies a higher priority. We assume that the ratio of QoS priorities is $\rho_{1}: \rho_{2}: \rho_{3}: \rho_{4}$.

\subsection{Single request case $(r=1)$}

We first consider the case that the number of requests for a subchannel is one. Let $P_{b}$ denote the probability that a certain bit is marked as 1 . Then, $P_{b}$ is given by

$$
P_{b}=\frac{\rho_{1} l_{1}+\rho_{2} l_{2}+\rho_{3} l_{3}+\rho_{4} l_{4}}{m} .
$$

The false positive probability is influenced by the spectral sidelobes problem [28] in OFDM-based systems as well as the inherent false positive property (i.e., logical false positive) of Bloom filters. The spectral sidelobes problem [28] refers to that a subcarrier may accidentally be set to 1 because of the leakage of power from nearby subcarriers. Therefore, the spectral leakage is considered in deriving the false positive probability. Let $P_{\text {leak }}$ be the probability of the spectral leakage, and we assume that the only two adjacent subcarriers cause power leakage. Then, the probability that a certain tagged bit is set to 1 due to the spectral leakage from two adjacent bits is given by $2 P_{\text {leak }}-2 P_{b} P_{\text {leak }}$, where we subtract the probability $2 P_{b} P_{\text {leak }}$ of the event that both the two adjacent bits are set to 1 and they simultaneously affect the tagged bit due to the spectral leakage. Thus, the probability that a certain bit is set to 1 , denoted by $P_{\text {positive, }}^{1}$ is as follows:

$$
P_{\text {positive }}^{1}=P_{b}\left(1+2 P_{\text {leak }}-2 P_{b} P_{\text {leak }}\right) .
$$


Then, the probability that the bit is set to 0 is given by $1-P_{\text {positive }}^{1}$

\subsection{Multiple requests case $(r>1)$}

Next, we extend to the case of multiple requests onto a subchannel. If there are $r$ requests to a subchannel, the probability that a certain bit is not set to 1 is by any of the hash functions is given as $P_{\text {negative }}^{r}=\left(1-P_{\text {positive }}^{1}\right)^{r}$. Similarly, the probability that the bit is set to 1 is $P_{\text {positive }}^{r}=$ $1-\left(1-P_{\text {positive }}^{1}\right)^{r}$. Then, we can derive the false positive probability that the AP incorrectly considers that a certain STA has requested for a given subchannel although the STA actually has not requested for the subchannel. The probability for a signature of length $l$ is obtained as:

$$
P_{\text {falsepositive }}^{D-F i}=\left(P_{\text {positive }}^{r}\right)^{l} \text {. }
$$

since it is the case that all of the $l$ signature bits assigned to the STA are set to 1 .

\subsection{Discussions}

False positive may cause the waste of wireless channels, since it can make the QoS-AP to wrongly allocate corresponding subchannels to the STA that has not requested the subchannels and thus the falsely allocated channels will remain unused until the next channel allocation. Note that the false positive does not always lead to the false subchannel allocations, since the falsely recognized request might not be accepted by the AP, particularly in the case of the false positive for low priority requests. However, the false positive for signatures with higher priority will likely suffer from the false channel allocation for the request resulting in the channel underutilization - because the AP basically grants more channel access opportunity to the STAs that have high priority packets.

Therefore, it is important to carefully determine the system parameters for QoS provisioning, i.e., lengths of signatures $\left(l_{i}\right)$. We consider the following to choose proper values of $l_{i}$.

- Although a longer signature has a lower false positive probability, it may suffer from the decrease in the channel quality estimation accuracy. This is because the total transmission energy spreads evenly over each of $l$ bits marked as 1 when transmitting a

Q-CRQ symbol. To address this tradeoff, we set the maximum length of a signature to 16 , i.e., the Q-CRQ symbol energy is spread at most to the quarter of a subchannel, so that the channel estimation error is maintained below $1 \mathrm{~dB}$.

- The difference in length among the four QoS types of signatures, i.e., $l_{i}-l_{i+1}, i \in\{1,2,3\}$, affects the false positive probability as well. If we set the difference in signature length between two adjacent QoS levels to be big, it makes the traffic with a higher priority have a very low false positive probability at the cost of the false probability of the lower priority traffic. This may be seen as the debate of QoS differentiation. For a given target QoS differentiation degree, the proper values of signature lengths can be derived. In this work, we apply the exponential decay in setting the signature, that is, we set the lengths for four levels of signatures, $l_{1}=16, l_{2}=8, l_{3}=6$, and $l_{4}=4$. Consequently, the highest priority traffic has a very low false positive rate.

Figures 4 and 5 show the false positive probabilities of QoS-Fi for two different scenarios as a function of the number of requests for a subchannel per a channel contention, with $P_{\text {leak }}=0.1$ and $m=64$. Figure 4 represents the case that traffic is evenly distributed throughout the levels of priority $\left(\rho_{1}: \rho_{2}: \rho_{3}: \rho_{4}=0.25: 0.25: 0.25:\right.$ $0.25)$, while Figure 5 represents the case that the network is dominated by high priority traffic $\left(\rho_{1}: \rho_{2}: \rho_{3}: \rho_{4}=\right.$ $0.4: 0.2: 0.2: 0.2$ ). As anticipated, the false positive probability of QoS-Fi increases as the number of requests for a subchannel increases. Especially, when the number of requests becomes large, QoS-Fi suffers from high false positives, which is a conventional issue caused when using a Bloom filter ${ }^{\mathrm{f}}$.

We can also observe that the false positive probability of each QoS priority level request is affected by the signature lengths as well as the ratio of QoS priorities given by offered load. We empirically have observed that the set of parameters, i.e., $l_{1}=16, l_{2}=8, l_{3}=6, l_{4}=4$, yields an acceptable false positive rate throughout various traffic load conditions; thus this parameter set is used for our simulations.

\section{Performance evaluation}

\subsection{Implementation}

\subsubsection{QoS-Fi prototype and experiment setup}

We prototyped the QoS-Fi OFDM-based PHY/MAC on a small testbed of four USRP nodes [33] each with GNU software define radio (SDR) [34]. We used the XCVR2450 daughterboard that uses transmissions near the $2.4 / 5 \mathrm{GHz}$ frequency band. We employ BAM that modulates each bit by on-off signaling for use of each Q-CRQ and Q-CRP frame.

We adaptively configure the signal power level comparison threshold to minimize the false positive rate of the subcarrier-level signal detection as follows. We first assign a bit pattern for each STA, then the combined signal (from all the STAs) must be the bitwise OR of all the bit patterns assigned. Given a threshold value, this combined signal can be converted into a binary bit sequence. Comparing this converted bit sequence with the (computed) bitwise OR of all the bit patterns assigned, the AP 

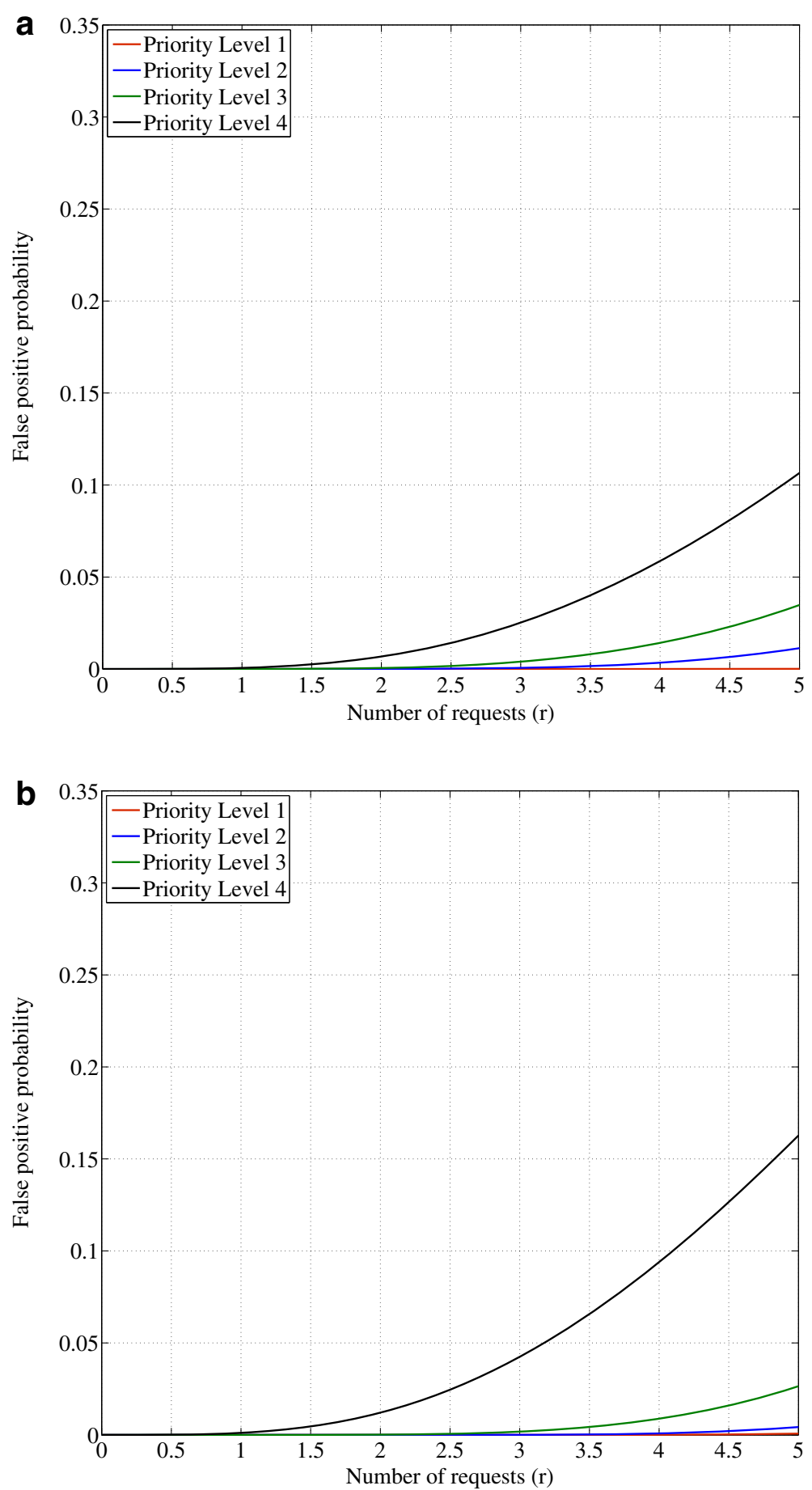

Figure 4 False positive probability of QoS-Fi. Under the evenly distributed traffic $\left(\rho_{1}: \rho_{2}: \rho_{3}: \rho_{4}=0.25: 0.25: 0.25: 0.25\right)$, with spectral leakage $P_{\text {leak }}=0.1$, number of subcarrier $m=64$. (a) The lengths of signatures $\left(l_{i}\right)$ are $I_{1}: I_{2}: l_{3}: I_{4}=16: 8: 6: 4$. (b) The lengths of signatures $\left(l_{i}\right)$ are $I_{1}: I_{2}: I_{3}: I_{4}=16: 12: 8: 4$

can determine whether the threshold value is the appropriate set. Adjusting threshold values, the AP searches the threshold value that yields the smallest error probabilities. In practice, this adaptation is triggered when a new STA joins the network or wrong Q-CRQ decoding results are frequently produced.

The experiments were conducted at a indoor laboratory (Figure 6a) to show the feasibility of the QoS-Fi 

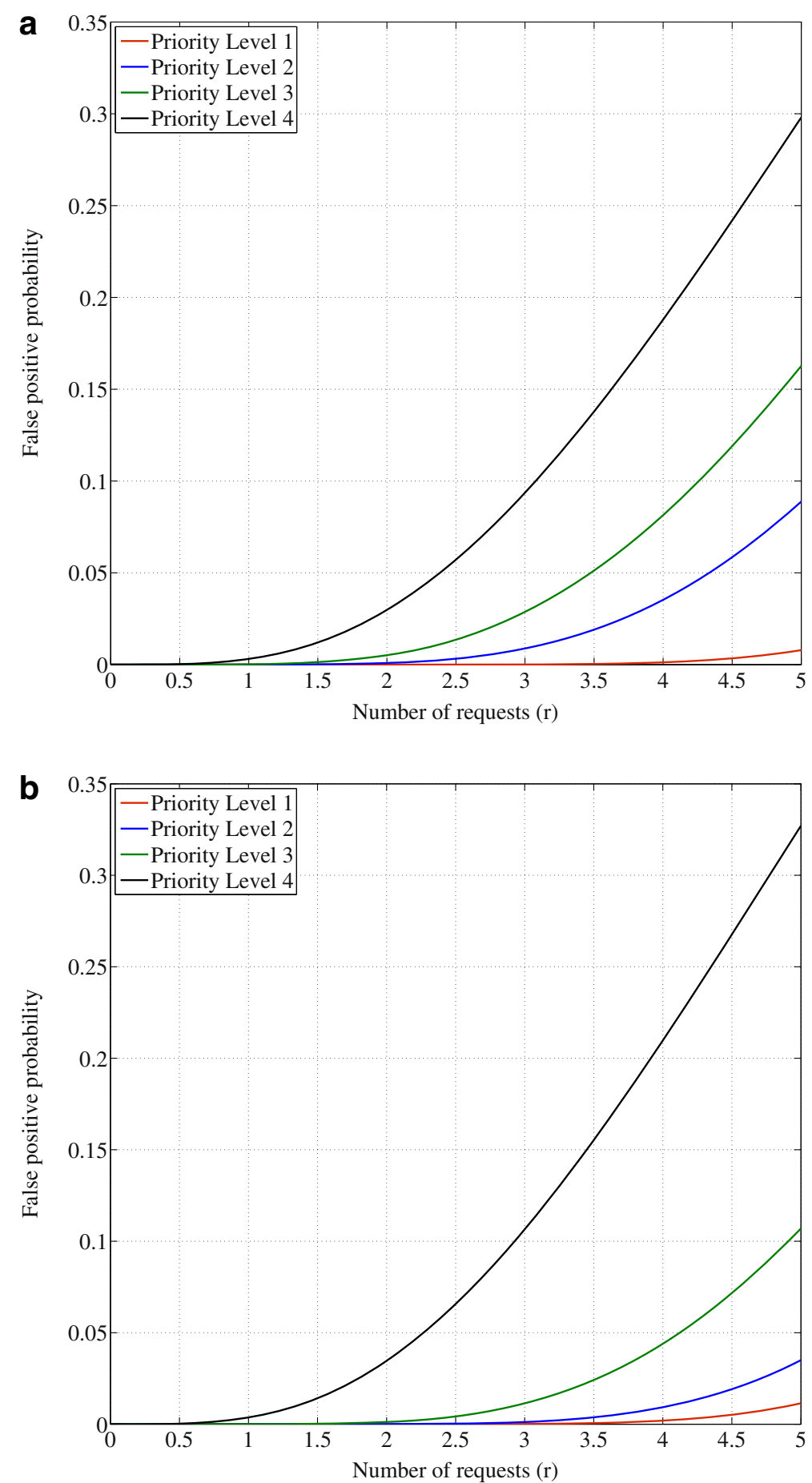

Figure 5 False positive probability of QoS-Fi under the scenario that the network is dominated by high priority traffic. With spectral leakage $P_{\text {leak }}=0.1$, number of subcarrier $m=64$. (a) The lengths of signatures $\left(l_{i}\right)$ are $I_{1}: l_{2}: l_{3}: I_{4}=16: 8: 6: 4$. (b) The lengths of signatures $\left(l_{i}\right)$ are $I_{1}: I_{2}: I_{3}: I_{4}=16: 12: 8: 4$.

PHY/MAC protocol real wireless networks. The QoS-Fi protocol implementation in the USRP node is shown in Figure $6 \mathrm{~b}$. We choose four positions randomly, and let one node serve as a QoS-Fi AP and the other three nodes as
QoS-Fi STAs associated with the AP. A rich set of the TX powers provided by the USRP/GNURadio is used, resulting up to $10 \mathrm{~dB}$ difference between the min and max received signal strengths at a given topology. 


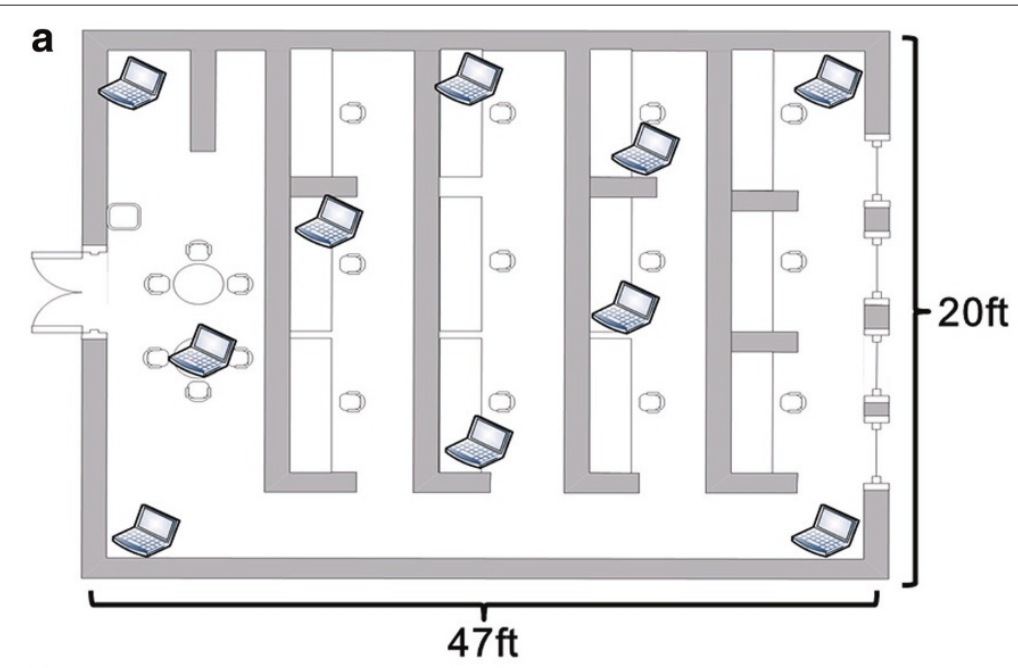

b

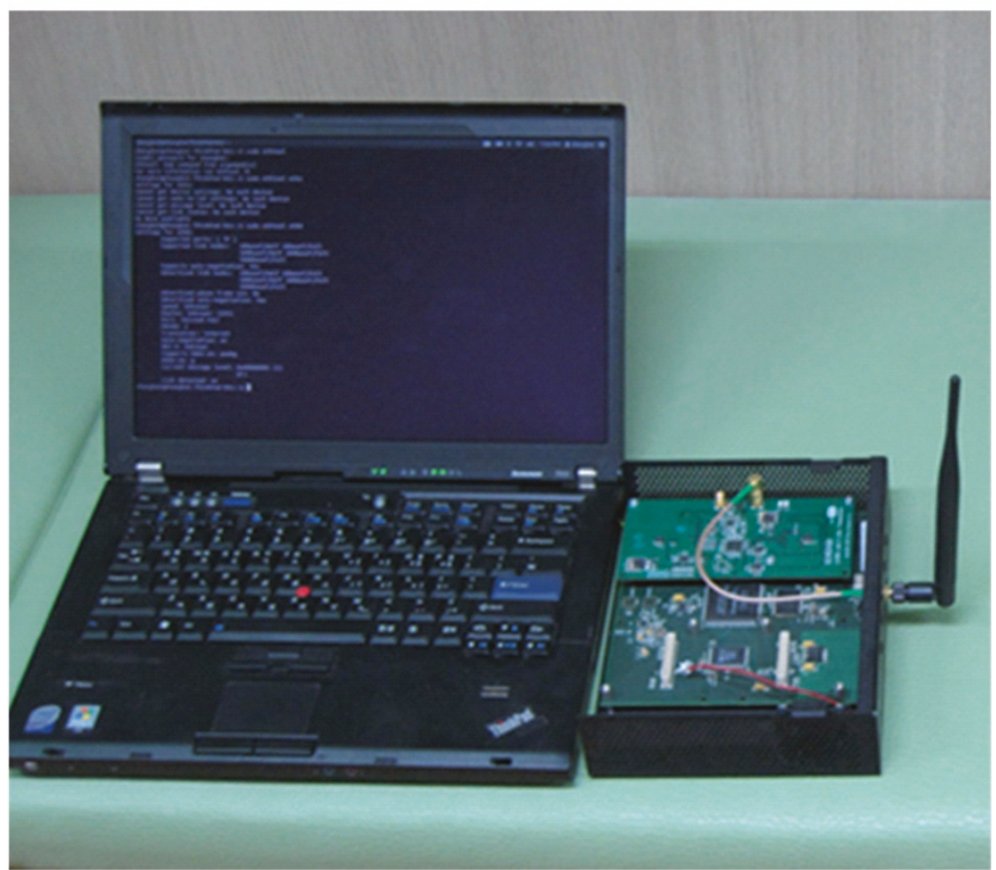

Figure 6 Experimental setup - the topology and the USRP platform. (a) The topology used in our experiment. (b) The QoS-Fi implementation on the USRP.

\subsubsection{Experimental results}

Figure 7 depicts the experimental results. In our experiments, multiple Q-CRQ symbols are combined at the receiver, since three STAs simultaneously transmit the QCRQ symbol. The SNR of this combined Q-CRQ symbol, measured at the receiver, is plotted along the $x$-axis. Let $P_{i}, i \in\left\{\mathrm{STA}_{1}, \mathrm{STA}_{2}, \mathrm{STA}_{3}\right\}$ be the signal strength of the individual Q-CRQ symbol transmitted from $\mathrm{STA}_{i}$. We call the case that satisfies the condition $\max \left\{\mathrm{STA}_{i}-\mathrm{STA}_{j}\right\}<$ $5 d B, i \neq j$ 'similar case' and otherwise 'different case'. The experimental results show the bit-wise false positive and negative rates for various degrees of the received signal strength. Overall, both false positive and negative rates are close to zero when the proper threshold is set.

Typically, for 'similar case', the threshold value found from our adaptive threshold setup is $3 \sim 5 \mathrm{~dB}$ lower than the SNR of the combined Q-CRQ symbol, and for 'different case', the value is $5 \sim 8 \mathrm{~dB}$ lower than the SNR of the combined Q-CRQ symbol. When the combined signal itself exhibits very low SNRs, careful adaption is required, which results in the threshold 


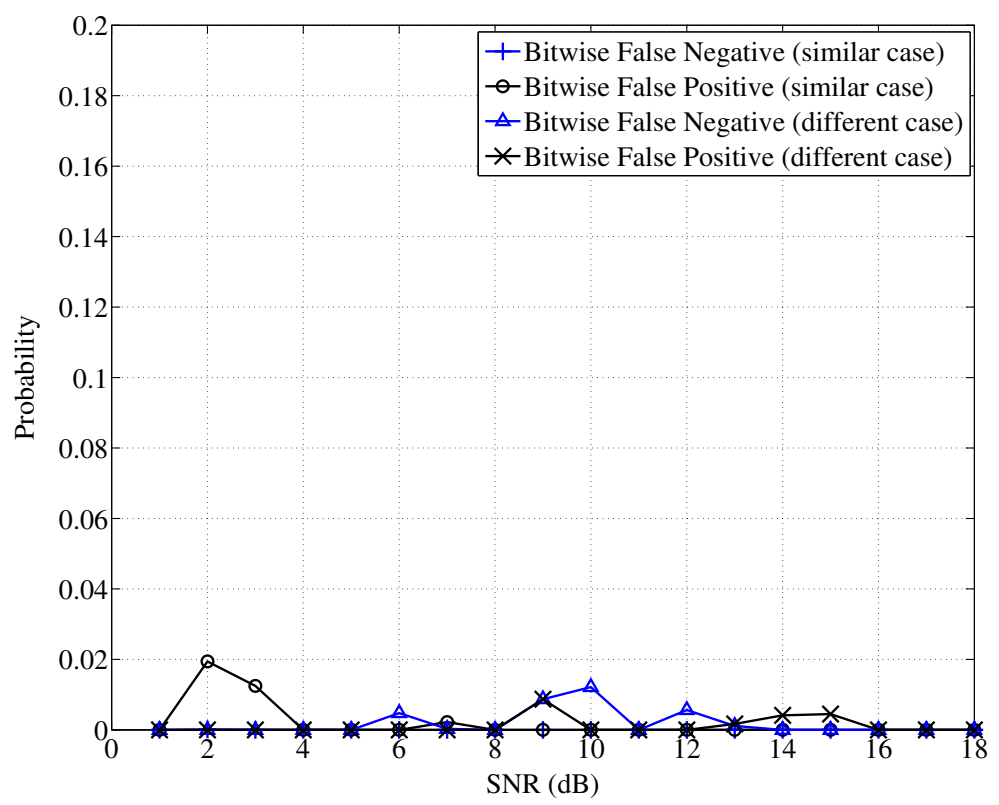

Figure 7 The accuracy of the subcarrier-level signal detection.

value close to $0 \mathrm{~dB}$ lower than the SNR of the combined Q-CRQ symbol (hence, cannot be quantified at $\mathrm{dB}$ scale). Even so, in the whole range of our experiment setups, the QoS-Fi's subcarrier-level signaling performs reliably.

Next, we show the accuracy of our channel estimation method. As shown in Figure 3, for most of the cases ( $\geq 90 \%$ ), the estimation error is less than or equal to $1 \mathrm{~dB}$. These two results show that the QoS-Fi's channel contention and estimation mechanisms are practically feasible in typical indoor environments.

\subsection{Trace-driven simulation}

\subsubsection{Simulation setup}

The results in the QoS-Fi USRP prototype show that the Bloom filter-based channel contention and estimation method is feasible in the real wireless network. However, with USRP experimentations, it is difficult to show how well QoS-Fi exploits the frequency diversity and provides QoS. In USRP, the signal processing is mainly conducted at the software; thus, it is very limited to process a narrow-band signal. In contrast, the Wi-Fi generally uses a wide-band $(20 \mathrm{MHz}$ or more) signal. In addition, the supported data rate is not as high as that in hardware radios at the current development stage of USRP. Therefore, we resort to tracedriven MATLAB simulations to assess the performance of QoS-Fi.

To conduct high fidelity emulation of real-world settings, we have used the $802.11 \mathrm{n}$ data traces provided by the authors of [6]. The traces are obtained from commodity Intel Wi-Fi Link $5300 \mathrm{NIC}$ and its modified driver [35]. The traces contain per-subcarrier (30 subcarriers for $20 \mathrm{MHz}$ ) RSSI readings for both the 24 mobile and 30 static diverse links. With the 54 diverse links, we set up 50 nodes in our simulations. We assume that time is slotted, and each node receives QoS traffic with the ratio of $\rho_{i}$ (depending on simulation scenarios) from the upper layer queues. The offered load follows the Poisson distribution, with the arrival rate of $\lambda$. The best-performing system parameters are empirically found to assess the best performance of QoS-Fi. As a result, we used $\alpha=0.1, \beta=2$, and $K=3$ in the simulations.

\subsection{Compared schemes}

We compare the performance of QoS-Fi with the following schemes. Note that we modified all the previous schemes to use a subchannel as an access basis for fair comparison.

- EDCA, the time-domain QoS support mechanism in WLANs. The EDCA protocol provides a distributed QoS provisioning function. Compared to other centralized algorithms, it cannot guarantee the performance of each QoS traffic.

- $\mathrm{K} \& H / R R$ [22], the state-of-the-art scheme designed for cellular networks. Unlike EDCA, this provides the statistical QoS guarantee (formally defined with effective capacity) using the admission control, hence is classified as a centralized resource allocation strategy. K\&H/RR combines two well-known channel 
allocation strategies, i.e., $\mathrm{K} \& H$ and $R R$ with the pre-defined ratio.

- The maximum throughput unit, the maximum capacity under the given offered load. This is given as the theoretical upper bound in terms of throughput and hence achieves the maximum Shannon's capacity of wireless channels. However, it is QoS-oblivious.

\subsection{Simulation scenarios}

To assess the performance of QoS-Fi in various environments, we conduct the simulation in the following scenarios.

- Scenario 1: Evenly distributed traffic throughout the QoS types $\left(\rho_{1}: \rho_{2}: \rho_{3}: \rho_{4}=1: 1: 1: 1\right)$ and moderate offered load $(\lambda=0.2)$.
- Scenario 2: Background traffic is twice as large as other types of traffic, $\left(\rho_{1}: \rho_{2}: \rho_{3}: \rho_{4}=1: 1: 1: 2\right)$ and moderate offered load $(\lambda=0.2)$.

- Scenario 3: Evenly distributed traffic throughout the QoS types $\left(\rho_{1}: \rho_{2}: \rho_{3}: \rho_{4}=1: 1: 1: 1\right)$ and high offered load $(\lambda=0.5)$.

- Scenario 4: Background traffic is twice as large as other types of traffic, $\left(\rho_{1}: \rho_{2}: \rho_{3}: \rho_{4}=1: 1: 1: 2\right)$ and high offered load $(\lambda=0.5)$.

\subsubsection{Simulation results}

System throughput. Figure 8 shows the overall aggregate throughput of QoS-Fi compared to the other schemes. We observe that QoS-Fi outperforms other QoS-aware schemes in terms of aggregate throughput in all the scenarios, where the throughput gains over EDCA and
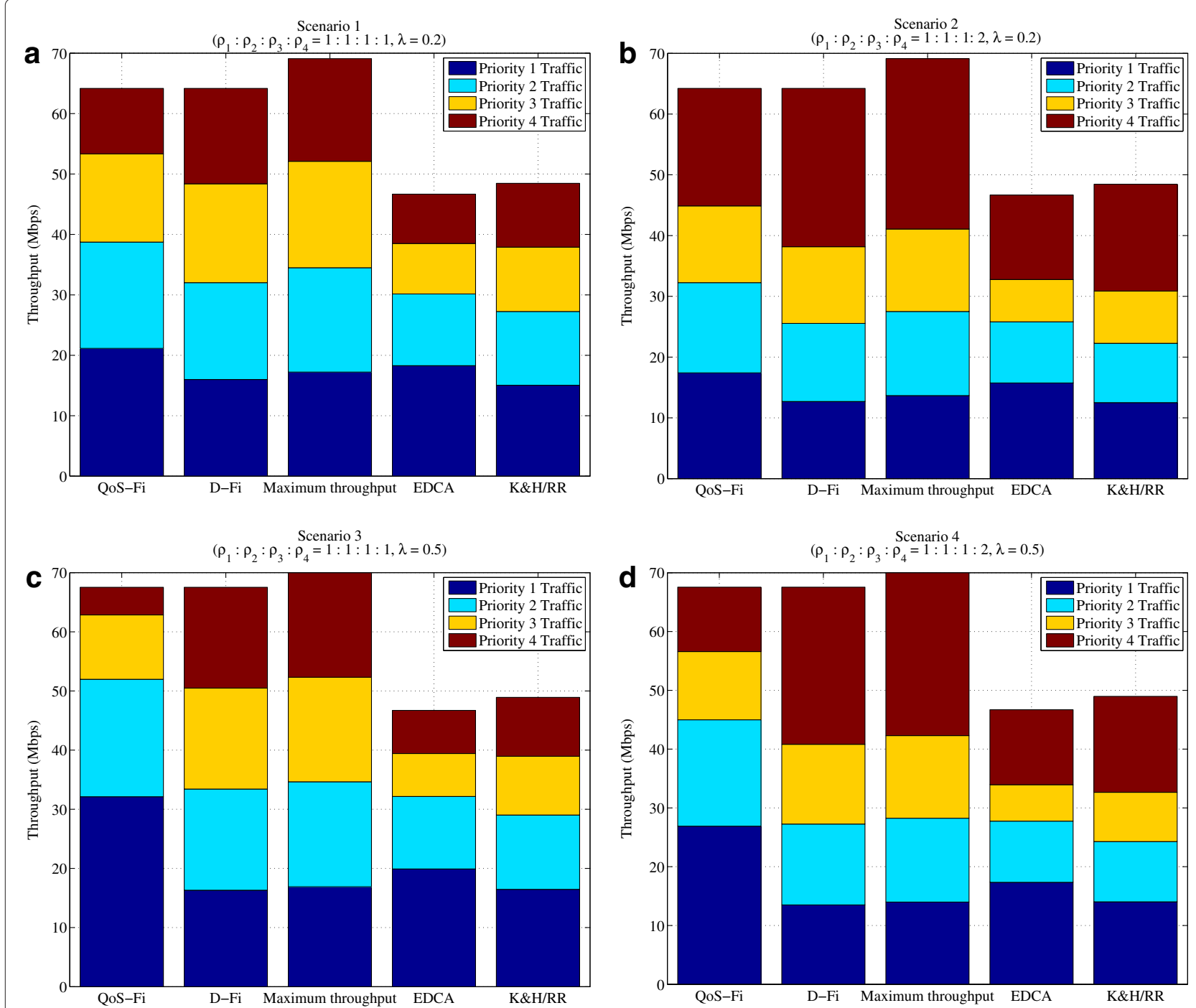

Figure 8 Average throughput per QoS priority level (a-d). 
$\mathrm{K} \& H / R R$ are up to $1.39 \mathrm{x}$ and $1.29 \mathrm{x}$, respectively. In addition, QoS-Fi achieves $93.5 \%$ of the maximum Shannon's wireless capacity (represented by Maximum throughput), which shows that QoS-Fi harnesses frequency diversity while still providing QoS. The reasons are (i) QoSFi jointly achieves the frequency diversity gains and QoS-awareness, since both are conducted in the frequency domain. The other QoS-aware schemes such as EDCA are frequency-oblivious and cannot exploit the frequency diversity gains, and (ii) K\&H/RR considers both frequency-diversity and QoS-awareness like QoS-Fi, however, it does not efficiently amortize MAC overhead, hence the maximum achievable throughput is upper bounded by the MAC inefficiency.

Average throughput per QoS priority level. Figure 8 also shows the QoS differentiation capability of QoS-Fi, against the other QoS-oblivious and QoS-aware schemes. Additional to the overall system throughput, we plot the average throughput for each QoS type for each scheme. The results imply the followings:

- Although our QoS provision mechanism comprises the decentralized feature (the EDCA-like distributed

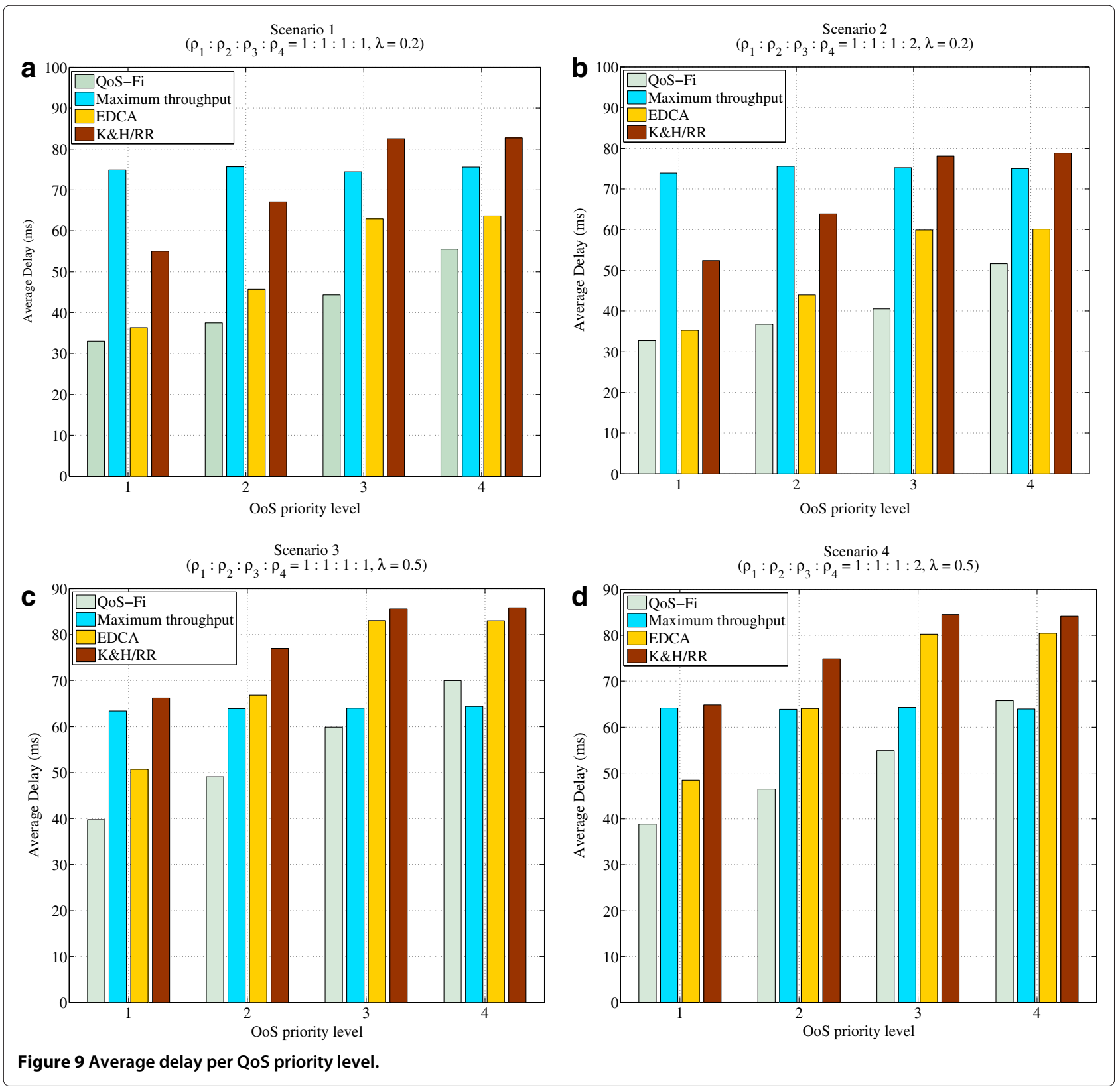


QoS channel contention) and centralized feature (the AP-side admission control), the degree of QoS-differentiation of QoS-Fi is most substantial, when compared with the other QoS-aware schemes. Moreover, the degree of QoS differentiaion can be flexibly further tuned by varying the parameter settings.

- By comparing the scenario of moderate offered load and the scenario of high offered load (i.e., Figure 8a,b versus Figure 8c,b), the degree of QoS-differentiation of QoS-Fi is larger in high offered load conditions. This is because the MAC layer queue holds the previously untransmitted (by channel contention) packets, until the new higher priority packets arrive at the queue; higher priority packets preempts the lower priority ones, depriving the lower priority packets of the opportunity to participate channel contention. This is more frequent in the high offered load scenarios, resulting in higher degree of QoS-differentiation in the QoS-Fi scheme.

- QoS differentiation is also observed in other QoS aware schemes. However, due to the QoS-Fi's frequency diversity awareness plus the MAC efficiency, QoS-Fi yields much higher system throughput. Contrary to QoS-aware schemes, the maximum throughput is QoS-oblivious and hence provides no QoS prioritization under all the scenarios.

- QoS-Fi achieves comparable performance with our previous scheme, D-Fi [7] in terms of aggregate throughput. However, we observe that QoS-Fi additionally provides the QoS-functionality under all the scenarios.

Average MAC delay. Figure 9 shows the average MAC delay, defined the time duration from the event that a packet is arrived at the MAC layer to the event that the packet is received to the receiver, for all the schemes. Again, the simulations are conducted in the aforementioned four scenarios. The results imply the followings:

- As anticipated, overall, average MAC delay for the QoS-Fi scheme is the shortest among all the compared schemes. We observe that the QoS-awareness is achieved by all the schemes, except for the maximum throughput scheme which is QoS-oblivious.

- For QoS-differentiation, higher priority packets have higher probabilities to be selected than the lower priority packets and experience lower MAC delay. Further, the MAC delay is also a function of TXbitrate and thus QoS-Fi outperforms MAC-inefficient QoS schemes such as EDCA and K\&H/RR.

\section{Conclusions}

We presented QoS-Fi, a novel QoS provisioning WiFi PHY/MAC protocol based on the OFDM technique. The proposed protocol efficiently exploits frequency diversity to satisfy the diverse QoS requirements of heterogeneous real-time mobile users, with minimum channel estimation and contention coordination cost. We showed the feasibility of QoS-Fi by implementing it on a USRP/GNUradio testbed. We also showed that the QoS-Fi PHY/MAC protocol can efficiently exploit frequency diversity while meeting the diverse QoS requirements, with real trace driven MATLAB simulations.

\section{Endnotes}

${ }^{a}$ Note that there are various ways to generate necessary signatures.

${ }^{\mathrm{b}}$ We assume that each hash function chooses a different bit for the sake of the convenience in the mathematical analysis.

'For the sake of simplicity, we note the signature of length 2; however, there are signatures of other lengths that can be falsely decoded in the variable length Bloom filter.

${ }^{\mathrm{d}}$ Here, we hypothesize that the multiplicative decrease (MD) feature of the AIMD algorithm enables QoS-Fi to react to the intensive channel variation, e.g., fast channel fluctuation and/or growth of contention level in particular channels. It is well known that the AIMD algorithm is a feedback control widely used for performing resource allocation operating in a distributed manner (whose convergence to the optimal operational point has been proved in [36]). However, we do not provide the mathematical or empirical grounds for this hypothesis as it is out of the scope of this paper. For interested readers, see [37] that deals with this issue.

${ }^{\text {e}}$ Signatures assigned to the associated STAs generated a set of hash functions, where each hash function selects each binary bit with equal probability.

${ }^{f}$ In our previous work [7], we have presented two approaches to address this problem. Please see [7] for details of how we have mitigated false positives. However, our main focus in this paper is the QoS provisioning mechanism.

\section{Competing interests}

The authors declare that they have no competing interests.

\section{Acknowledgements}

This work was supported in part by the IT R\&D program of MSIP/KEIT. [10041861, Development of Wired/Wireless i-AVB System Technology for Concurrent Transmission of HD Media and Control Data], and the National Research Foundation of Korea(NRF) Grant funded by the Korean Government(MSIP)(No. 2012R1A1A1014755, 2013R1A1A1006823). 


\section{Author details}

${ }^{1}$ Department of Computer Science, Seoul National University, Seoul, Korea. ${ }^{2}$ Department of Software Design and Management, Gachon University, Seongnam, South Korea.

Received: 21 November 2013 Accepted: 29 August 2014 Published: 15 September 2014

\section{References}

1. A Goldsmith, Wireless Communications. (Cambridge University Press, 2005)

2. IEEE Std. 802.16-2004, IEEE Standard for Local and Metropolitan Area Networks, Part 16: Air Interface for Fixed broadband Wireless Access Systems. (Std. IEEE)

3. 3GPP TS 36.201-820, Evolved Universal Terrestrial Radio Access (E-UTRA); Long Term Evolution (LTE) Physical Layer; General Description. 3GPP

4. D Halperin, W Hu, A Sheth, D Wetherall, Predictable 802.11 packet delivery from wireless channel measurements. ACM SIGCOMM Comput. Commun. Rev. 41(4), 159-170 (2011)

5. H Rahul, F Edalat, D Katabi, CG Sodini, Frequency-aware rate adaptation and MAC protocols, in Proceedings of the 15th Annual International Conference on Mobile Computing and Networking (MobiCom'09): Sept 20-25, 2009: Beijing (ACM, 2009), pp. 193-204

6. A Bhartia, Y-C Chen, S Rallapalli, L Qiu, Harnessing frequency diversity in multicarrier wireless networks, in Proceedings of the 17th Annual International Conference on Mobile Computing and Networking (MobiCom'11): Sept 19-23, 2011; Las Vegas (ACM, 2011), pp. 253-264

7. SLee, C-K Kim, D-Fi: A diversity-aware Wi-Fi using an OFDM-based Bloom filter, in Network Protocols (ICNP) 2012 20th IEEE International Conference On: Oct. 30, 2012 to Nov. 2, 2012 (IEEE, 2012), pp. 1-10

8. IEEE Std. 802.11n-2009, IEEE Standard for Local and Metropolitan Area Networks, Part 11: Wireless LAN Medium Access Control (MAC) and Physical Layer (PHY) Specifications Amendment 5: Enhancements for Higher Throughput. Std. IEEE

9. Y Shen, E Martinez, Channel Estimation in OFDM Systems. (Application Note, Freescale Semiconductor, 2006)

10. H Kwon, S Kim, BG Lee, Opportunistic multi-channel CSMA protocol for OFDMA systems. Wireless Commun. IEEE Trans. 9(5), 1552-1557 (2010)

11. KTan, J Fang, Y Zhang, S Chen, L Shi, J Zhang, Y Zhang, Fine-grained channel access in wireless LAN. ACM SIGCOMM Comput. Commun. Rev. 41(4), 147-158 (2011)

12. S Sen, R Roy Choudhury, S Nelakuditi, No time to countdown: migrating backoff to the frequency domain, in Proceedings of the 17th Annual International Conference on Mobile Computing and Networking (MobiCom'11): Sept 19-23, 2011; Las Vegas (ACM, 2011), pp. 241-252

13. X Feng, J Zhang, Q Zhang, B Li, Use your frequency wisely: explore frequency domain for channel contention and ACK, in Proceedings of the 31st Annual IEEE International Conference on Computer Communications (INFOCOM'12): Mar. 25-30; Orlando (IEEE, 2012), pp. 549-557

14. Y Lu, B Prabhakar, F Bonomi, Bloom filters: design innovations and novel applications, in Proc. of the 43rd Annual Allerton Conference: Sept 28-30; Monticello (IEEE, 2003)

15. KWu, H Li, L Wang, Y Yi, Y Liu, D Chen, X Luo, Q Zhang, LM Ni, hJam: attachment transmission in WLANs. Mobile Comput. IEEE Trans. 12(12), 2334-2345 (2013)

16. J Huang, F Qian, Y Guo, Y Zhou, Q Xu, ZM Mao, S Sen, O Spatscheck, An in-depth study of LTE: effect of network protocol and application behavior on performance, in Proceedings of the ACM SIGCOMM 2013 Conference on SIGCOMM: Aug 12-16, 2013 Hong Kong (ACM, 2013), pp. 363-374

17. H Yang, F Ren, C Lin, J Zhang, Frequency-domain packet scheduling for 3GPP LTE uplink, in Proceedings of the 29th Annual IEEE International Conference on Computer Communications (INFOCOM'10): Mar 15-19; San Diego (IEEE, 2010), pp. 1-9

18. Y Zhang, SC Liew, Proportional fairness in multi-channel multi-rate wireless networks - part II: the case of time-varying channels with application to OFDM systems. Wireless Commun. IEEE Trans. 7(9), 3457-3467 (2008)

19. GS Moya, JZ Flores, Parameters of a $2.4 \mathrm{GHz}$ wide band radio channel for WLAN applications, in Electronics, Communications and Computers, 2004. CONIELECOMP 2004. 14th International Conference On: Feb 16-18, 2004; Veracruz (IEEE, 2004), pp. 41-44
20. H MacLeod, C Loadman, Z Chen, Experimental studies of the $2.4 \mathrm{GHz}$ ISM wireless indoor channel, in Communication Networks and Services Research Conference, 2005. Proceedings of the 3rd Annual: May 16-18, 2005; Halifax (IEEE, 2005), pp. 63-68

21. Q Liu, S Zhou, GB Giannakis, Cross-layer scheduling with prescribed QoS gurantees in adaptive wireless networks. Selected Areas Commun. IEEE J. 23(5), 1056-1066 (2005)

22. D Wu, R Negi, Downlink scheduling in a celluar network for quality of service assurance. Vehic. Technol. IEEE Trans. 53(5), 1547-1557 (2004)

23. IEEE Std. 802.11 e-2005, IEEE Standard for Local and Metropolitan Area Networks, Part 11: Wireless LAN medium access control (MAC) and physical layer (PHY) Specifications Amendment 8: Medium Access Control (MAC) Quality of Service enhancements. Std. IEEE

24. A Broder, M Mitzenmacher, Network applications of Bloom filters: a survey. Internet Math. 1(4), 485-509 (2004)

25. STarkoma, CE Rothenberg, E Lagerspetz, Theory and practice of Bloom filters for distributed systems. Commun. Surv. Tutor. IEEE. 14(1), 131-155 (2012)

26. M Liu, M Crussiere, J Helard, Improved channel estimation methods based on pn sequence for tds-ofdm, in Telecommunications (ICT), 2012 19th International Conference On: April 23-25, 2012; Jounieh (IEEE, 2012), pp. 1-5

27. D Chu, Polyphase codes with good periodic correlation properties (corresp.) Inform. Theory IEEE Trans. 18(4), 531-532 (1972)

28. HA Mahmoud, H Arslan, Sidelobe suppression in OFDM-based spectrum sharing systems using adaptive symbol transition. Commun. Lett. IEEE. 12(2), 133-135 (2008)

29. G Bianchi, I Tinnirello, Kalman filter estimation of the number of competing terminals in an IEEE 802.11 network, in Proceedings of the 23rd Annual IEEE International Conference on Computer Communications (INFOCOM'03): Mar 30-April 3 2003; Orlando, vol. 2 (IEEE, 2003), pp. 844-852

30. Z-Q Luo, S Zhang, Dynamic spectrum management: complexity and duality. Selected Topics Signal Process. IEEE J. 2(1), 57-73 (2008)

31. D-H Shin, S Bagchi, C-C Wang, Distributed online channel assignment toward optimal monitoring in multi-channel wireless networks, in INFOCOM, 2012 Proceedings IEEE (IEEE, 2012), pp. 2626-2630

32. S Li, L Cai, A distributed optimization framework for multi-channel multi-user small cell networks. Commun. Lett. IEEE (2014)

33. Etturs Research. http://www.ettus.com

34. GNU Radio Project. http://gnuradio.org/redmine/projects/gnuradio/wiki

35. D Halperin, W Hu, A Sheth, D Wetherall, Tool release: gathering $802.11 \mathrm{n}$ traces with channel state information. ACM SIGCOMM Comput. Commun. Rev. 41(1), 53-53 (2011)

36. D-M Chiu, R Jain, Analysis of the increase and decrease algorithms for congestion avoidance in computer networks. Comput. Netw. ISDN Syst. 17(1), 1-14 (1989)

37. S Lee, J Choi, J Yoo, C-K Kim, Frequency diversity-aware Wi-Fi using OFDM-based Bloom filters. Mobile Comput. IEEE Trans. (preprint) (2014)

doi:10.1186/1687-1499-2014-152

Cite this article as: Lee et al:: Providing quality-of-service for

frequency-aware Wi-Fi using OFDM-based variable-length Bloom filters.

EURASIP Journal on Wireless Communications and Networking 2014 2014:152.

\section{Submit your manuscript to a SpringerOpen ${ }^{\circ}$ journal and benefit from:}

- Convenient online submission

- Rigorous peer review

- Immediate publication on acceptance

- Open access: articles freely available online

- High visibility within the field

- Retaining the copyright to your article

Submit your next manuscript at $\gg$ springeropen.com 